\title{
Retraction
}

\section{Author-Initiated Retraction: Li et al., Elevation of Brain Magnesium Prevents and Reverses Cognitive Deficits and Synaptic Loss in Alzheimer's Disease Mouse Model}

At the request of the authors, The Journal of Neuroscience is retracting "Elevation of Brain Magnesium Prevents and Reverses Cognitive Deficits and Synaptic Loss in Alzheimer's Disease Mouse Model” by Wei Li, Jia Yu, Yong Liu, Xiaojie Huang, Nashat Abumaria, Ying Zhu, Xian Huang, Wenxiang Xiong, Chi Ren, Xian-Guo Liu, Dehua Chui, and Guosong Liu, which appeared on pages 8423-8441 of the May 8, 2013 issue. The authors report, "This article described the effects of elevating brain magnesium on preventing and reversing cognitive deficits in an Alzheimer's disease mouse model. During recent efforts to extend this work, we discovered errors in the quantification of the expression and/or phosphorylation of a subset of signaling pathways, particularly related to Figures 4 and $5 D$. Despite these errors, the major conclusions of the paper remain substantiated. As any correction will require substantial rewriting of the manuscript, we ask to withdraw the article. A corrected treatment will be published in the future. We apologize for any confusion caused by this error."

DOI:10.1523/JNEUROSCI.1265-14.2014 